\title{
Complicaciones Asociadas Al Trauma Penetrante de Esófago, Experiencia De 11 Años En El Hospital Universitario de Neiva
}

\author{
${ }^{1}$ Maria Alejandra Rubio, ${ }^{2}$ Juan Jose Rubio, ${ }^{3}$ Manuel Alejandro Ospitia, ${ }^{4} J u a n$ Felipe Sanjuan \\ ${ }^{5}$ Rolando Medina, ${ }^{6}$ Wilmer Fernando Botache
}

\section{RESUMEN}

Antecedentes: Las lesiones penetrantes en el esófago son extremadamente raras, menos del $0.5 \%$; se relacionan con alta morbilidad $30-66 \%$ y mortalidad $\sim 20 \%$; existe un desafío diagnóstico y terapéutico; dada su severidad se requiere un abordaje oportuno y un manejo agresivo para evitar secuelas y complicaciones.

Diseño del estudio: Análisis descriptivo de subgrupo de una cohorte retrospectiva (2003-2013) de pacientes con trauma quirúrgico. La severidad del trauma fue evaluada mediante la clasificación AAST.

Resultados: Del total 2390 casos de trauma quirúrgico registrados, $10(0.4 \%)$ corresponden a trauma de esófago. Todos los casos fueron observados en hombres con una mediana de edad de 26 años $(R I C=19-35)$. La causa de la lesión más frecuente fue la herida por arma de fuego en 6 pacientes, seguido de herida por arma corto punzante en 2 pacientes y de onda expansiva en los 2 pacientes restantes. EI RTS en la mayoría de pacientes fue de $7.84(\mathrm{RIC}=7.47-7.84)$. La severidad del trauma esofágico fue grado II en 7 pacientes y grado III en 3 pacientes. Se observaron lesiones vasculares asociadas en 2 pacientes. Todos los pacientes fueron llevados a reparo primario de la lesión. La complicación más frecuente encontrada fue la fistula traqueo esofágica en 4 pacientes. 7 pacientes requirieron re intervención, $71 \%$ por lesiones relacionadas directamente a la lesión esofagica. No se presentó mortalidad.

Conclusiones: Las lesiones esofágicas en trauma de manejo quirúrgico son infrecuentes, no se observaron muertes en esta serie. Las complicaciones son habituales, la más comúnmente relacionada es la fistula traqueo esofágica, las reintervenciones presentaron adecuada evolución.

Significancia Clinica: La lesión esofágica es una lesión sumamente infrecuente con complicaciones importantes e incluso fatales, con esta investigación se busca conocer la epidemiologia relacionada con dichas lesiones, conocimiento que es clave en el proceso de aprendizaje para ofrecer mejores y mas efectivos abordajes en el momento de verse enfrentado a un trauma esofágico.

Palabras claves: Fistula traqueo esofágica, gastrostomía, Trauma esofágico, traqueostomía.

\footnotetext{
${ }^{1}$ Medica General ${ }^{2}$ Medico Interno, ${ }^{3}$ Medico General ${ }^{4}$ Residente de Cirugia General ${ }^{5}$ Cirujano General ${ }^{6}$ Cirujano de Trauma y Emergencias

${ }^{1-6}$ Universidad Surcolombiana, Neiva, Huila, Colombia

Corresponding Author: Maria Alejandra Rubio, Medica General, Universidad Surcolombiana, Neiva, Huila, Colombia Phone: +5788718310, e-mail: alerubi3@gmail.com
}

\section{ABSTRACT}

Background: Penetrating esophageal lesions are extremely rare, less than $0.5 \%$. They are related to high morbidity $30--66 \%$ and mortality $\sim 20 \%$. There is a diagnostic and therapeutic challenge, given its severity requires a timely approach and aggressive management to avoid sequelae and complications.

Study design: Descriptive subgroup analysis of a retrospective cohort (2003--2013) of patients with surgical trauma. The severity of the trauma was assessed using the AAST classification.

Results: Of the total 2,390 cases of registered surgical trauma, $10(0.4 \%)$ corresponded to an esophageal trauma. All cases were observed in men with a median age of 26 years (IQR = 19--35). The cause of the most frequent injury was gunshot injury in six patients, followed by blunt gun injury in two patients and blast wave in the two remaining patients. The RTS in the majority of the patients was 7.84 (IQR = 7.47--7.84). The severity of esophageal trauma was grade II in seven patients and grade III in three patients. Associated vascular lesions were observed in two patients. All patients were taken to repair the primary lesion. The most frequent complication was esophageal tracheal fistula in four patients. A total of seven patients required reintervention, $71 \%$ for lesions directly related to esophageal lesion. No mortality was shown.

Conclusion: Esophageal lesions in surgical management trauma are infrequent, no deaths were observed in this series. Complications are common, the most commonly related is tracheoesofagic fistula, the reinterventions present an adequate evolution

Keywords: Esophageal trauma, Tracheoesofagic fistula, Tracheostomy, gastrostomy.

How to cite this article: Rubio MA, Rubio JJ, Ospitia MA, Sanjuan JF, Medina R, Botache WF. Complicaciones Asociadas Al Trauma Penetrante de Esófago, Experiencia De 11 Años En El Hospital Universitario de Neiva. Panam J Trauma Crit Care Emerg Surg 2017;6(1):25-29.

Source of support: Nil

Conflict of interest: None

\section{INTRODUCCION}

El trauma continúa siendo la primera causa de mortalidad en menores de 40 años, los accidentes, actos delincuenciales y violencia corresponden a las principales etiologías con una alta carga de morbilidades y secuelas para los sobrevivientes. La región cervical contiene órganos vitales en una relativamente pequeña y sin protección región anatómica, siendo una de las localizaciones más

This paper was presented in Student Research Competition in PTS Annual Congress 2015, Santa Cruz, Bolivia 
vulnerables del cuerpo, como región le concierne el 10\% de las localizaciones de trauma ${ }^{1}$ en especial las lesiones que involucran la Zona II; Las lesiones cervicales por ubicación, extensión a órganos complejidad, son un desafío quirúrgico una alta morbimortalidad.

Las lesiones esofágicas son extremadamente infrecuentes, aproximadamente $<0,5 \%$ en las series por trauma general, comprometiendo mayormente el esófago en su porción cervical $~ 82 \%,{ }^{2}$ aunque, la mayoría de las complicaciones se asocian a su porción torácica; ${ }^{3}$ su cercanía a estructuras vitales circulatorias y respiratorias le confieren una alta morbimortalidad convirtiendo dichas lesiones en una situación clínica potencialmente mortal que requiere un abordaje preciso y rápido para su corrección.

Excluyendo las causas de perforación esofágica espontanea (51\%) o iatrogénica (33\%), ${ }^{4}$ en el contexto de trauma, el estado de inconsciencia y las lesiones concomitantes no permiten la mejor evaluación clínica ni su diagnóstico inmediato, incluso con un paciente alerta la semiología de las lesiones esofágicas no es muy precisa, los signos y síntomas pueden ir desde dolor cervical hasta enfisema subcutáneo pasando por disfagia y disfonía entre otros. Existen métodos diagnósticos radiológicos e invasivos para confirmar el diagnóstico de perforación, pero, su uso depende de la disponibilidad en cada centro de atención, se considera la tomografía computarizada como un método confiable ${ }^{5}$ aunque el esofagograma con medio de contraste hidrosoluble sumado a la esofagoscopia pueden tener una sensibilidad hasta del $100 \%$ en hallar perforaciones esofágicas ${ }^{6}$

El abordaje quirúrgico se prefiere por cervicotomia izquierda por la lateralización del esófago a nivel cervical, se puede utilizar rafia a 1-2 planos y azul de metileno para corroborar integridad es practicado en diferentes grupos quirúrgicos. Aunque se describen casos exitosos donde se puede considerar un manejo conservador con antibióticos y apoyo nutricional con resultados igualmente favorables. ${ }^{7}$

La mortalidad históricamente podría ser tan alta que alcanzaba hasta un $80 \%,{ }^{8}$ pero, con las nuevas alternativas diagnosticas se ha logrado un abordaje más oportuno con una disminución significativa de la mortalidad $(\sim 20 \%),{ }^{9,10}$ aunque, con complicaciones y morbilidades de hasta un $40-60 \%^{111,12}$ se ha demostrado incluso, que la mortalidad no cambia cuando se hace diagnostico preoperatorio de la lesión esofágica no obstante con disminución de gastos hospitalarios, ${ }_{1}^{13}$ las presentaciones tardías y una corrección de manera no inmediata conduce a peores resultados.

Ha de esperarse una tendencia en los resultados similar a las estadísticas internacionales en términos de morbimortalidad, se presume en el contexto de Colombia como país con altos índices de violencia y trauma, y adicionalmente, que el estudio fue realizado en un hospital de III nivel y centro de referencia de toda la región Surcolombiana.

\section{MATERIALES Y METODOS}

- Tipo de estudio: Cohorte Retrospectiva

- De acuerdo a la naturaleza de las variables se realizaron las medidas de resumen:

- Para las variables continuas se evaluó la normalidad de la distribución con la prueba de SaphiroWilk, cuando no se rechace la hipótesis de nulidad (Ho = Distribución normal) se utilizó la media y la desviación estándar como medidas de resumen. Cuando se rechazó de la hipótesis de nulidad $(\mathrm{Ha}=$ No corresponde a una distribución normal) se utilizó la mediana y el rango intercuartilico como medidas de resumen.

- Para las variables categóricas: Se resumieron mediante las frecuencias absolutas acompañado de su frecuencia relativa.

- Consideraciones clínicas: la severidad de las lesiones y el compromiso de las áreas anatómicas se calificaron de acuerdo a las áreas anatómicas.

\section{DISEÑO DEL ESTUDIO}

Análisis descriptivo de subgrupo de una cohorte retrospectiva (2003-2013) de pacientes con trauma quirúrgico. La severidad del trauma fue evaluada mediante la clasificación AAST.

\section{CONSIDERACIONES ETICAS}

Se realizó un estudio de tipo retrospectivo de carácter descriptivo, no se realizó ningún tipo de intervención, se utilizó como fuente primaria las historias clínicas, previo consentimiento del comité de ética del Hospital Universitario Hernando Moncaleano Perdomo. Clasificación de riesgo según la ley colombiana: Investigación sin riesgo (Resolución 8430 de 1993). No se tiene ningún conflicto de interés.

\section{RESULTADOS}

Del total 2390 casos de trauma quirúrgico registrados, $10(0.4 \%)$ corresponden a trauma de esófago. Todos los casos fueron observados en hombres con una mediana de edad de 26 años (RIC=19-35). La causa de la lesión más frecuente fue la herida por arma de fuego en 6 pacientes, seguido de herida por arma corto punzante en 2 pacientes y de onda expansiva en los 2 pacientes restantes. El RTS en la mayoría de pacientes fue de 7.84 (RIC= 7.47-7.84). La severidad del trauma esofágico fue grado II en 7 pacientes y grado III en 3 pacientes. Se observaron lesiones vasculares asociadas en 2 pacientes. (Tabla 1) 


\begin{tabular}{ll}
\hline \multicolumn{2}{c}{ Tabla 1. Caracteristicas de la poblacion } \\
\hline Variable & $n(\%)$ \\
\hline Edad (años) ${ }^{*}$ & $26(19-35)$ \\
Hombres & $10(100)$ \\
Mecanismo De Lesion & \\
HPAF & $6(60.0)$ \\
HACP & $2(20.0)$ \\
Onda Expansiva & $2(20.0)$ \\
Severidad Aast & \\
II & $7(70.0)$ \\
III & $3(30.0)$ \\
Lesion asociada & \\
Vascular & $2(20.0)$ \\
Tráquea & $5(50.0)$ \\
\hline *MED (RIC) &
\end{tabular}

Todos los pacientes fueron llevados a reparo primario de la lesión utilizando rafia primaria como abordaje quirúrgico. La complicación más frecuente encontrada fue la fistula traqueo esofágico en 4 pacientes. 7 pacientes requirieron reintervención que incluyeron $71 \%$ complicaciones relacionadas con el trauma de esófago directamente; con adecuada evolución posterior a la reintervencion. No se presentó mortalidad. En la tabla 2, se hace la caracterización de las lesiones y así mismo la descripción de las complicaciones.

\section{DISCUSION}

Según Delos Reyes y cols. ${ }^{14}$ En su serie de casos se reporta que el trauma de esófago es de etiología multifactorial, muy ocasionales y con alta morbilidad como ocurre en nuestro estudio.

Las lesiones esofágicas son extremadamente infrecuentes con desafíos clínico-quirúrgicos asociados a alta morbimortalidad, la aparición de complicaciones es representativa $46 \%$ según Makhani y cols. ${ }^{15}$ Nuestros resultados no muestran mortalidad y tenemos complicaciones en el $70 \%$. Es posible considerar que se debe vigilar y optimizar la técnica quirúrgica con sus cuidados postoperatorios; comparando los escritos consultados en Mattox y cols. ${ }^{16}$ La morbilidad alcanza un $~ 66 \%$ indiferente del tipo de complicación, lo que alcanza un estimado contemporáneo a nuestro estudio.

El trauma de esófago con perforación puede presentarse por iatrogenia o procedimientos endoscópicos como lo reporta Brinster y cols. ${ }^{17}$ Los resultados obtenidos en nuestra serie de casos no se encontraron por lesiones esofágicas por iatrogenias y evidenciamos que todas fueron por mecanismos externos.

Las complicaciones y la severidad del trauma están directamente relacionados con el mecanismo del trauma, según Ivatury y cols. ${ }^{18}$ Las heridas por arma de fuego se

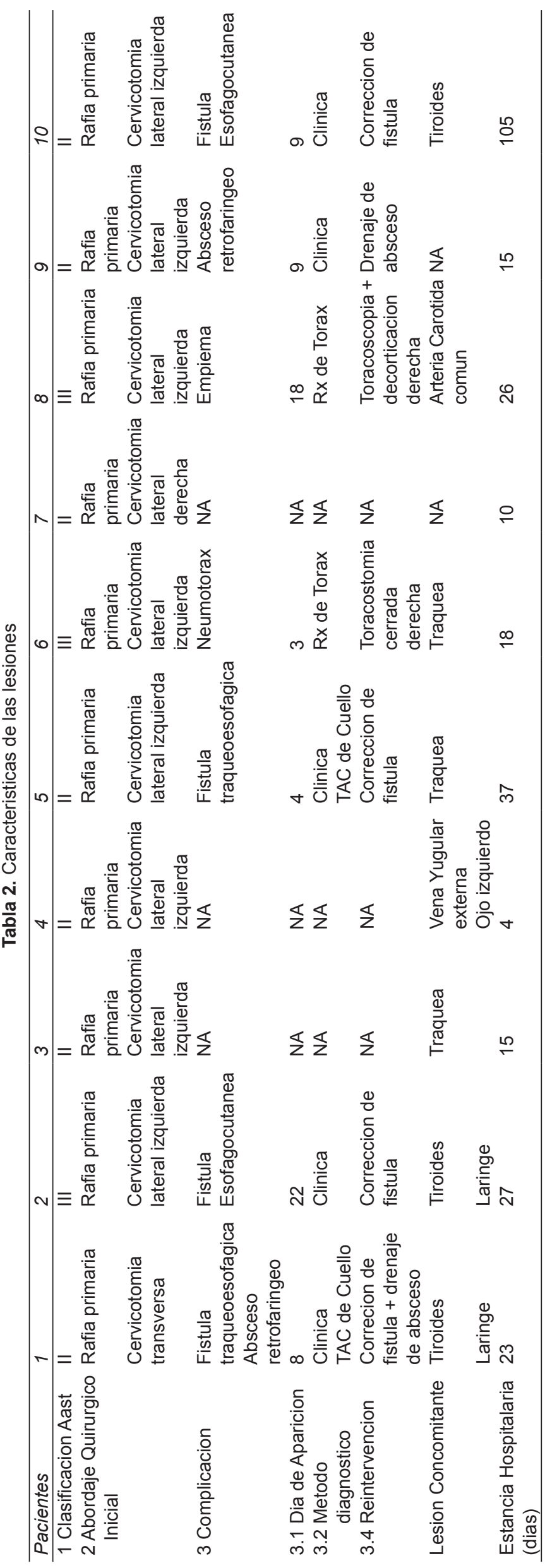


correlacionan con peores resultados, lesión de órganos concomitantes y aparición de fistulas, desenlaces que son similares a nuestros resultados teniendo las heridas por arma de fuego como el principal factor de riesgo para morbilidades.

El estudio de Carrott y cols. ${ }^{19}$ Presenta los objetivos del tratamiento en el trauma de esófago que corresponden a controlar un foco séptico, el cierre de la perforación y el drenaje de la contaminación asociada, sin embargo reporta una $20 \%$ de mortalidad. Nuestros resultados muestran que se realiza el manejo quirúrgico de limpieza local, esofagorrafia primaria y desenlace de mortalidad cero.

La reparación por esofagorrafia primaria corresponde a la primera opción en el abordaje de la perforación esofágica, conducta avalada por Johnson (2008), ${ }^{20}$ Mattox $(2013)^{21}$ y cols; en nuestro estudio se realiza manejos quirúrgicos tempranos, por la severidad de las lesiones y posibles complicaciones por lo anterior no se consideró el manejo conservador como lo sugiere De los Reyes y cols. ${ }^{22}$

\section{CONCLUSION}

Las lesiones esofágicas son extremadamente infrecuentes, en nuestra descripción se alcanzaron complicaciones hasta en un $60 \%$ de los pacientes, con requerimiento de re-intervención para corrección además de traqueostomía y gastrostomía, la principal complicación correspondió a la fistula traqueo-esofágica, las heridas por proyectil arma de fuego se relacionan con la aparición de complicaciones y no se presentó mortalidad, se debe considerar la creación de un protocolo para evitar la aparición de complicaciones.

\section{SIGNIFICANCIA CLINICA}

La lesión esofágica es una lesión sumamente infrecuente con complicaciones importantes e incluso fatales, con esta investigación se busca conocer la epidemiologia relacionada con dichas lesiones, conocimiento que es clave en el proceso de aprendizaje para ofrecer mejores y más efectivos abordajes en el momento de verse enfrentado a un trauma esofágico.

\section{REFERENCIAS}

1. Mahmoodie M, Sanei B, Moazeni-Bistgani M, Namgar M. Penetrating Neck Trauma: Review of 192 Cases. Arch Trauma Res 2012;1(1):14-18.

2. Ivatury RR, Moore F a, Biffl W, Leppeniemi A, Ansaloni L, Catena F, et al. Oesophageal injuries: Position paper, WSES, 2013. World J Emerg Surg. 2014;9(1):9.
3. Makhani M, Midani D, Goldberg A, Friedenberg FK. Pathogenesis and outcomes of traumatic injuries of the esophagus. Dis Esophagus. 2014 Sep-Oct;27(7):630-636.

4. Romero R V., Goh K-L. Esophageal perforation: Continuing challenge to treatment. Gastrointest Interv. Elsevier; 2013;2(1):1-6.

5. Conradie WJ, Gebremariam FA. Can computed tomography esophagography reliably diagnose traumatic penetrating upper digestive tract injuries? Clin Imaging. Elsevier B.V.; 2015;1-7

6. Mattox K, Moore E, Feliciano D. Trauma, Cap. Lung, Trachea, and Esophagus, 2013.Septima edición, p. 479-480

7. Delos Reyes AP, Clancy C, Lach J, Olorunto W a., Williams M. Conservative management of esophageal perforation after a fall. Int J Surg Case Rep. Surgical Associates Ltd; 2013;4(6):550-553.

8. Carrott PW, Low DE. Advances in the Management of Esophageal Perforation. Thorac Surg Clin. 2011;21(4):541-555.

9. Johnson SB. Esophageal Trauma. Semin Thorac Cardiovasc Surg.2008;20(1):46-51.

10. Mattox K, Moore E, Feliciano D. Trauma, Cap. Lung, Trachea, and Esophagus, 2013.Septima edición, p. 481

11. Makhani M, Midani D, Goldberg A, Friedenberg FK. Pathogenesis and outcomes of traumatic injuries of the esophagus. Dis Esophagus. 2014 Sep-Oct;27(7):630-636.

12. Mattox K, Moore E, Feliciano D. Trauma, Cap. Lung, Trachea, and Esophagus, 2013.Septima edición, p. 481

13. Yeh D, Hwabejire 2, de Moya M, King D, Fagenholz $P$, Kaafarani H, Klein E. Preoperative evaluation of penetrating esophageal trauma in the current era: An analysis of the National Trauma Data Bank. J Emerg Trauma Shock. 2015 Jan-Mar;8(1):30-33

14. Delos Reyes AP, Clancy C, Lach J, Olorunto W a., Williams $\mathrm{M}$. Conservative management of esophageal perforation after a fall. Int J Surg Case Rep. Surgical Associates Ltd; 2013;4(6):550-553.

15. Makhani M, Midani D, Goldberg A, Friedenberg FK. Pathogenesis and outcomes of traumatic injuries of the esophagus. Dis Esophagus. 2014 Sep-Oct;27(7):630-636.

16. Mattox K, Moore E, Feliciano D. Trauma, Cap. Lung, Trachea, and Esophagus, 2013.Septima edición, p. 481

17. Brinster CJ, Singhal S, Lee L, Marshall MB, Kaiser LR, Kucharczuk JC. Evolving options in the management of esophageal perforation. Ann Thorac Surg. 2004;77(4):1475-1483.

18. Ivatury RR, Moore F a, Biffl W, Leppeniemi A, Ansaloni L, Catena F, et al. Oesophageal injuries: Position paper, WSES, 2013. World J Emerg Surg. 2014;9(1):9.

19. Carrott PW, Low DE. Advances in the Management of Esophageal Perforation. Thorac Surg Clin. 2011;21(4):541-555.

20. Johnson SB. Esophageal Trauma. Semin Thorac Cardiovasc Surg.2008;20(1):46-51.

21. Mattox K, Moore E, Feliciano D. Trauma, Cap. Lung, Trachea, and Esophagus, 2013.Septima edición,p. 480

22. Delos Reyes AP, Clancy C, Lach J, Olorunto W a., Williams M. Conservative management of esophageal perforation after a fall. Int J Surg Case Rep. Surgical Associates Ltd; 2013;4(6):550-553. 


\section{Complicaciones Asociadas Al Trauma Penetrante de Esófago, Experiencia De 11 Años En EI Hospital Universitario de Neiva}

Despite current advancements in trauma surgery and damage control surgery, management of critically injured patients with penetrating trauma to the esophagus still conveys a high mortality. Due to the proximity of airway and major vessels, majority of deaths from penetrating esophageal injury occur during the first 24 hours of presentation. For this reason, early intervention is of the essence in this subset of patients. When diagnosis is made early, patient will need immediate primary closure as the recommended intervention. It is important for the surgeon to be suspicious and aware of patient deterioration in the clinical setting when esophageal injury is in doubt based on equivocal diagnostics.

Of interest, it is important to mention that the increased rate of esophageal related complications is associated with 3 key variables: extensive delay in diagnosis, patient age and AIS of the abdomen and pelvic contents $>3$. Regarding the nature of the esophageal injury, any esophageal injury AAST-OIS grade $>2$ and any complicated procedure such as resection or diversion increases the likelihood of esophageal related complications as well.

As a final note mortality from injury to the esophagus ranges from $7 \%$ to $27 \%$ and is often due to missed injuries, associated injuries, and septic complications. For this reason and based on multi-institutional studies rapid diagnosis and early repair should always be emphasized in patients with penetrating esophageal trauma. Complications from these injuries will result in prolong morbidity, overall increased hospital LOS and potentially late mortality.

Juan Duchesne MD, FACS, FCCP, FCCM Section Chief Trauma \& Critical CareTulane School of MedicineTICU Medical Director Norman E. McSwain Jr. MD Spirit of Charity Level I Trauma CenterUniversity Medical Center New Orleans

\section{Complicaciones Asociadas Al Trauma Penetrante de Esófago, Experiencia De 11 Años En EI Hospital Universitario de Neiva}

A pesar de los avances actuales en cirugía de trauma y cirugía de control de daños, el manejo de pacientes críticamente heridos por lesiones penetrantes del esófago aun expresa una alta mortalidad. Debido a la proximidad de las vías respiratorias y los vasos principales, la mayoría de las muertes por lesión esofágica penetrante ocurren durante las primeras 24 horas de presentación. Por esta razón, la intervención temprana es esencial en este subconjunto de pacientes. Cuando el diagnóstico se realiza tempranamente, el paciente necesitará el cierre primario inmediato como intervención recomendada. Es importante que el cirujano sea suspicaz y consciente del deterioro del paciente en el contexto clínico cuando la lesión esofágica sea dudosa basada en diagnósticos equívocos.

De interés, es importante mencionar que una tasa mayor de complicaciones relacionadas al esófago se asocia con 3 variables claves: retraso extenso en el diagnóstico, edad del paciente y AIS del abdomen y contenido pélvico $>3$. En cuanto a la naturaleza de la lesión esofágica, cualquier lesión esofágica con un grado de AAST-OIS > 2 y cualquier procedimiento complicado, como la resección o la desviación aumenta la probabilidad de complicaciones relacionadas al esófago.

Como nota final, la mortalidad por lesión del esófago oscila entre $7 \%$ y $27 \%$ y se debe a menudo a lesiones no identificadas, lesiones asociadas y complicaciones sépticas. Por esta razón y sobre la base de estudios multiinstitucionales, el diagnóstico rápido y la reparación temprana siempre deben enfatizarse en pacientes con lesiones esofágicas penetrantes. Las complicaciones de estas lesiones darán lugar a una morbilidad prolongada, un aumento general en la duración de hospitalización y la mortalidad potencialmente tardía.

Juan Duchesne MD, FACS, FCCP, FCCM Section Chief Trauma \& Critical CareTulane School of MedicineTICU Medical Director Norman E. McSwain Jr. MD Spirit of Charity Level I Trauma CenterUniversity Medical Center New Orleans 